\title{
MEASUREMENTS OF THE BEAM-BEAM INTERACTION AT PEP-II*
}

\author{
J. T. Seeman**, M. Biagini, Y. Cai, F. J. Decker, M. Donald, S. Ecklund, A. Fisher, R. Holtzapple, \\ R. Iverson, A. Kulikov, M. Minty, A. Novokhatski, I. Reichel, M. Sullivan, \\ J. Turner, U. Wienands, SLAC, Stanford, CA 94309, USA \\ C. Steier, M. Zisman, LBNL, Berkeley, CA 94720, USA \\ W. Kozanecki, Saclay, France
}

\begin{abstract}
The beam-beam interaction is one of the performance limitations at PEP-II. The peak luminosity in PEP-II has reached $3.4 \times 10^{33} / \mathrm{cm}^{2} / \mathrm{s}$ with 693 bunches with a positron current of $1.6 \mathrm{~A}$ and an electron current of $0.84 \mathrm{~A}$. The beam-beam tune shift limits have exceeded 0.06 horizontally and 0.03 vertically. A model of the luminosity with current using the measured beam sizes has been developed. Studies are underway to increase the luminosity toward $10^{34} / \mathrm{cm}^{2} / \mathrm{s}$ and beyond by increasing the number of bunches, raising the beam currents, lowering the betas at the interaction points, and shortening the bunch lengths[1].
\end{abstract}

\section{PEP-II CONDITIONS}

The peak PEP-II luminosity was achieved using 693 bunches with $1610 \mathrm{~mA}$ of positrons and $840 \mathrm{~mA}$ of

Table 1 PEP-II Collision Parameters as of July 21, 2001.

\begin{tabular}{|l|l|l|}
\hline IR Parameter & Design & $\begin{array}{l}\text { Present } \\
\text { operating }\end{array}$ \\
\hline C-M energy (GeV) & 10.58 & 10.58 \\
\hline Crossing angle (mrad) & 0 & $<0.1$ \\
\hline Luminosity (x10 ${ }^{33}$ ) & 3.0 & 3.40 \\
\hline Number of bunches & 1568 & 693 \\
\hline HER current (mA) & 750 & 840 \\
\hline LER current (mA) & 2140 & 1610 \\
\hline Beam-beam parameter (y+/-) & $0.03 / 0.03$ & $0.055 / 0.028$ \\
\hline Beam-beam parameter (x+/-) & $0.03 / 0.03$ & $0.069 / 0.059$ \\
\hline Bx*/ßy* (cm/cm) & $1.5 / 50$ & $1.15 / 50$ \\
\hline Optimum coupling (\%) & 3.0 & 2 to 6 \\
\hline Emittance (nm-rad) $\mathrm{x} / \mathrm{y}$ & $1.5 / 49$ & $3 / 30+, 2 / 50-$ \\
\hline IP beam sizes (microns) y/x & $4.7 / 157$ & $5 / 147$ \\
\hline Ey/x (microns) & $6.7 / 222$ & $6.7 / 207$ \\
\hline Injection top-off time (min) & 3 & 2 \\
\hline Injection full fill time (min) & 10 & 8 \\
\hline Detector solenoid field (T) & 1.5 & 1.5 \\
\hline Int. luminosity in 8 hr pb-1 & 45 & 76 \\
\hline Int. luminosity in 24 hr pb-1 & 135 & 220 \\
\hline Int. luminosity / week pb-1 & 785 & 1202 \\
\hline Int. luminosity / month & 3300 & 4200 \\
\hline Total Int. luminosity fb-1 & ------- & 40 \\
\hline
\end{tabular}

* Work support by US DOE contract DE-AC03-76SF00515.

**SEEMAN@SLAC.STANFORD.EDU electrons. Other parameters are shown in Table 1. The bunches were spaced every four RF buckets in 35 short trains of 20 bunches each. There is a 5\% ion clearing gap. The mini-gaps expel captured low energy e-. The beta $\mathrm{x}$ and $\mathrm{y}$ at the collision point were $50 \mathrm{~cm}$ and $1.25 \mathrm{~cm}$, respectively. The first $\mathrm{e}^{+}$bunch in each mini-train is under filled by about $10 \%$ to avoid blowing up its respective e bunch by the beam-beam force. That $\mathrm{e}^{+}$bunch is not enlarged as much by ECI as the other $\mathrm{e}^{+}$bunches[2,3]

\section{BEAM-BEAM TESTS}

Along the bunch train the luminosity shows a general variation which is caused by the relative LERHER RF phase shift along the train. See Figure 1 and 2. This variation was predicted in early project planning and the general slope can be compensated. The fast ripples along the train are a result of parked cavities that are

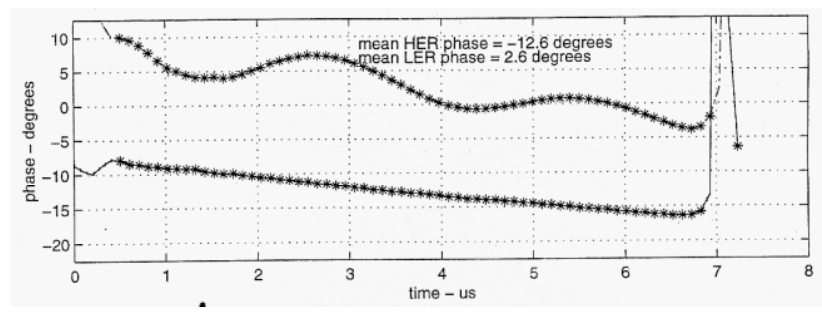

Figure 1. The variation of the bunch RF phase along the bunch train for the LER top and HER bottom. Note the three degrees relative variation in the LER.

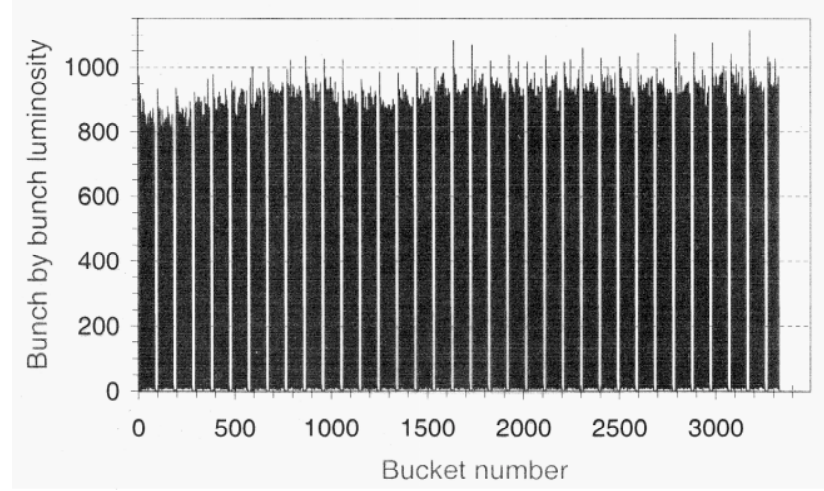

Figure 2. The measured luminosity in each bunch along the bunch train. Note that the luminosity dips where the LER RF phase has maxima. 


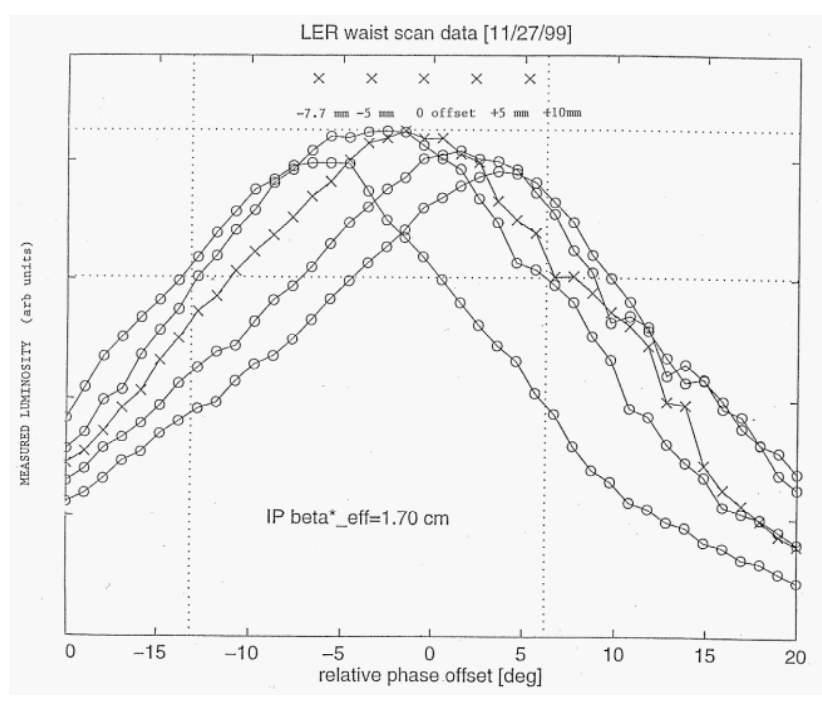

Figure 3. Variations in luminosity with HER RF phase as a function of location of the LER vertical beta waist.

not powered but soon will be.

As seen from these RF phase measurements, centering the vertical betas in the IR is very important. Tests were made to center the minima of the IR y beta functions at the collision point by moving the waists with the IR quadrupoles while measuring the luminosity using the $\mathrm{rf}$ relative phase. See Figure 3. The waists were found to be very nearly centered to a few $\mathrm{mm}$.

Several coasting beam fills with BaBar were tried with a bunch pattern having every other bucket filled. This bunch spacing is the design pattern (called the "by-2" pattern) having potential parasitic crossing effects. With full colliding currents and the by-2 bunch pattern the nominal peak luminosity was nearly achieved (90-95\%) in a few hours. The main effect was to shift the vertical tune by about 0.01 . This bodes well for the future for obtaining higher luminosity with more bunches.

The transverse sizes of the positron beam are enlarged at high currents by the electron cloud instability (ECI) from multipacting electrons, as is now commonly seen in several accelerators. A solenoid capable of 30 gauss has been wrapped on the straight section vacuum chambers to suppress these electrons. A length of $600 \mathrm{~m}$ has been wound resulting in a luminosity increase of order $70 \%$ over the past year. With the present solenoids the e+ beam size enlargement starts at about $1300 \mathrm{~mA}$ with four bucket spacing and is seen in both the vertical and horizontal planes. PEP-II is now embarking on a program to wind solenoids in the LER bending arcs to suppress multipacting and photo-electrons there. About 200 chambers ( $5 \mathrm{~m}$ each) need winding. $80 \mathrm{~m}$ is done so far.

The beams at the interaction point were measured to be tilted by about 2 degrees relative to the machine axis. Vertical beam-beam scans were performed as a function of relative $\mathrm{x}$ offset of the two beams and the vertical centers of the scans were plotted versus the $\mathrm{x}$ position to reveal the tilt. There were also hints from the out of plane beam-beam deflection scans that the two beams were slightly tilted relative to each other.

The luminosity was measured as a function of the product of the beam currents as shown in Figure 4. From the non-linearity, some of the beam sizes must increase with current. The HER electron beam sizes were measured as a function of the product of the beam currents as shown in Figure 5. The e- vertical size enlarges at low currents from the beam-beam effect. This continues until the electron cloud effect enlarges the positron beam as seen in Figure 7 and relaxes the beambeam forces on the HER electrons. The LER positron sizes continue to increase above $1300 \mathrm{~mA}$, thus, stopping the growth of the electron tune shifts as are shown in Figure 6. The positron tune shifts in Figure 8 continue to increase with current as the electron beam sizes are relatively constant with further current changes.

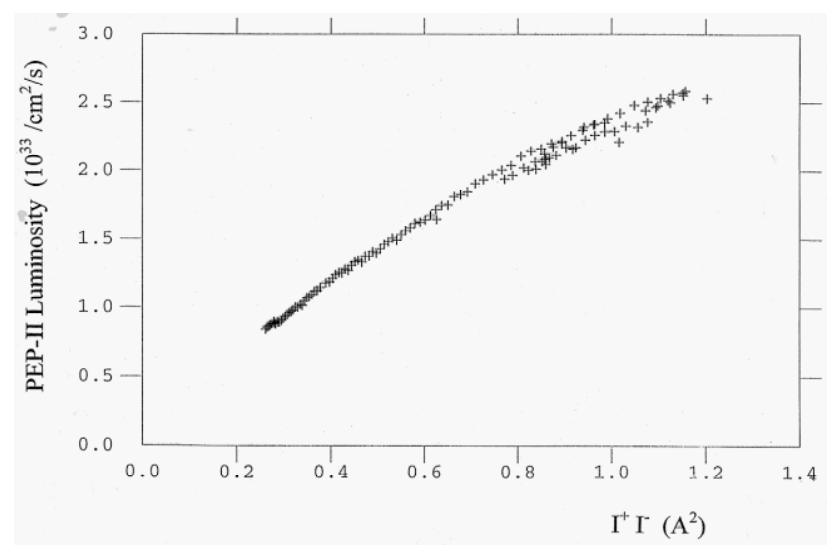

Figure 4. PEP-II luminosity versus the product of the beam currents. Note that the luminosity deviates from an ideal square law due to the enlargement of the beam sizes.

\section{CONCLUSIONS}

PEP-II is operating at a luminosity of $3.4 \times 10^{33} / \mathrm{cm}^{2} / \mathrm{s}$. Beam-beam effects are definitely seen but are sometimes masked by the electron cloud effect in the LER. The electron cloud enlarges the e+ beam starting about 1300 $\mathrm{mA}$. The derived tune shift values (limits) are 0.021 for evertical and 0.044 for e- horizontal and 0.046 for e+ vertical and 0.07 for e+ horizontal. Detailed size measurements have started which will help resolve subtle enlargement effects. Energy transparency conditions appear to be quite weak as PEP-II violates the ratio of beam currents, emittance ratios, beta* ratios and damping time equality but the luminosity and tune shifts are good.

\section{REFERENCES}

[1] J. Seeman et al, "PEP-II Status and Plans," Proceedings of PAC 2001.

[2] I. Riechel, et al, "Beam-Beam Simulations for PEPII," Proceedings of PAC2001.

[3] F-J. Decker et al, "PEP-II Bunch Patterns," Proceedings of PAC2001. 

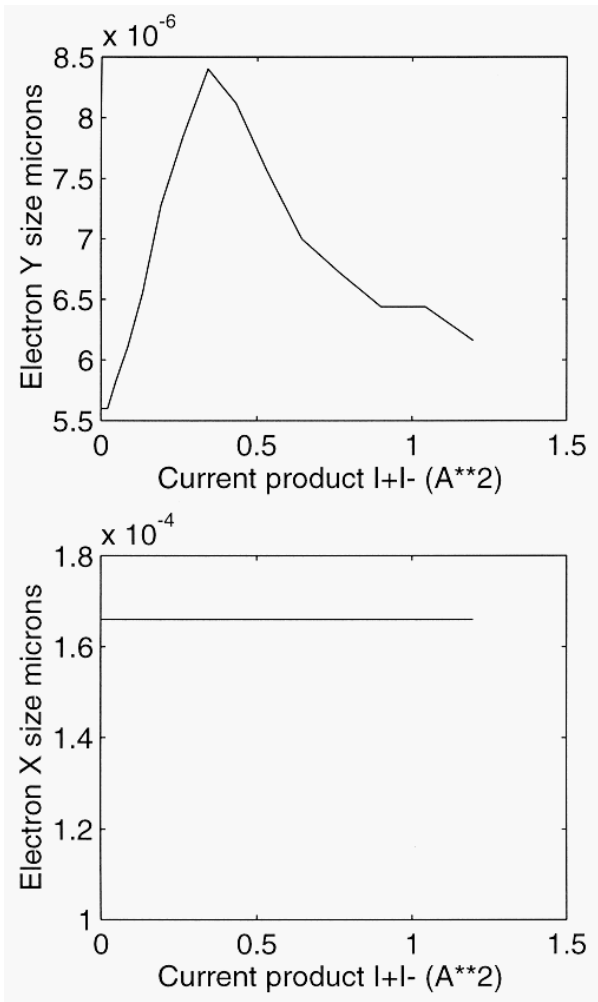

Figure 5. Measured HER electron beam sizes as a function of the product of the beam currents.
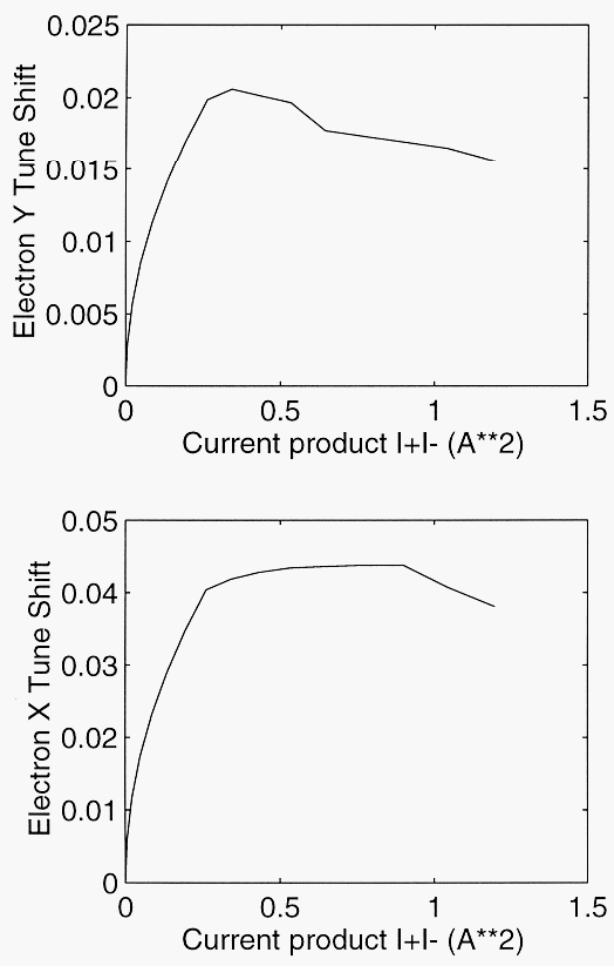

Figure 6. Derived beam-beam tune shift parameters versus current for HER electron.
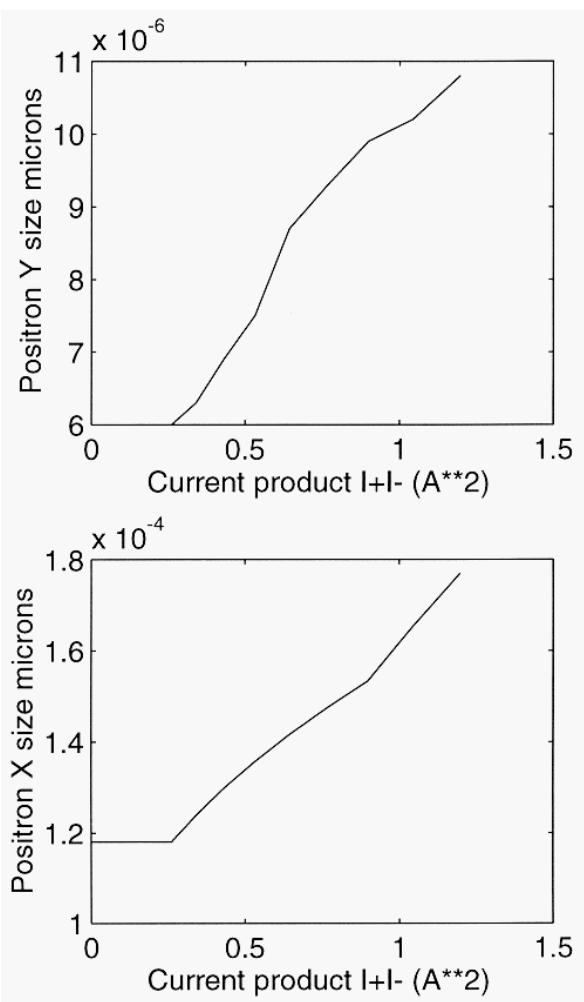

Figure 7. Measured LER positron beam sizes as a function of the product of the beam currents.
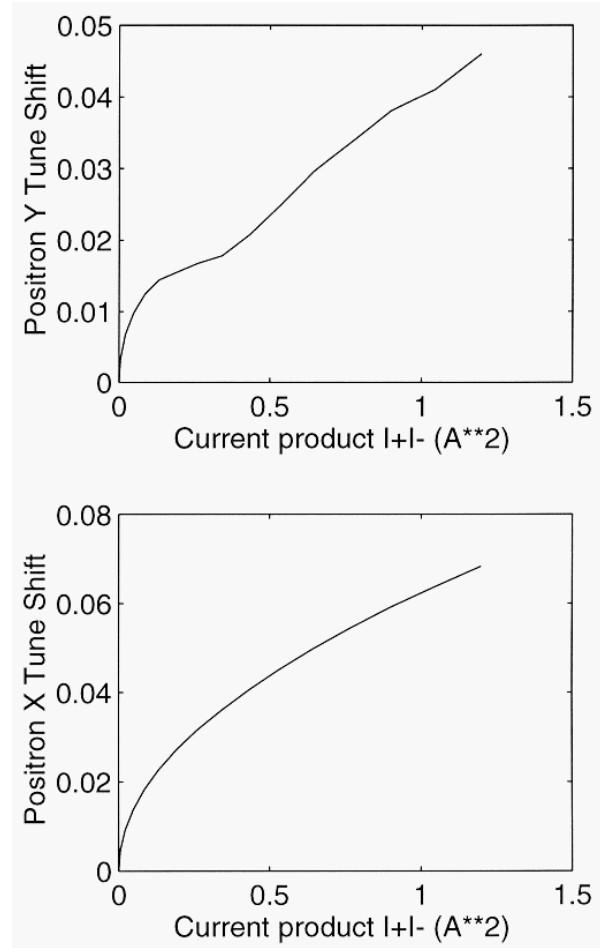

Figure 8. Derived beam-beam tune shift parameters versus current for LER positrons. 